DOI: 10.1002/aenm.201401778

Article type: Full Paper

\title{
Interplay Between Side-Chain Pattern, Polymer Aggregation, and Charge Carrier Dynamics in PBDTTPD:PCBM Bulk-Heterojunction Solar Cells
}

Clare Dyer-Smith, Ian A. Howard, Clément Cabanetos, Abdulrahman El Labban, Pierre M. Beaujuge, * Frédéric Laquai*

Dr. C. Dyer-Smith, Dr. I.A. Howard, Dr. F. Laquai

Max Planck Research Group for Organic Optoelectronics

Max Planck Institute for Polymer Research

Ackermannweg 10

D-55128 Mainz, Germany

E-mail: laquai@mpip-mainz.mpg.de

Dr. C. Cabanetos, Dr. A. El Labban, Prof. P.M. Beaujuge

Chemical Sciences, and Materials Science \& Engineering

Division of Physical Sciences \& Engineering

King Abdullah University of Science and Technology (KAUST)

Thuwal 23955-6900, Saudi Arabia

E-mail: pierre.beaujuge@kaust.edu.sa

Keywords: polymer solar cells, charge generation, transient absorption spectroscopy, structure-property relationships, PBDTTPD

PBDTTPD polymer donors with linear side-chains yield bulk-heterojunction (BHJ) solar cell power conversion efficiencies (PCE) of about $4 \%$ with $\mathrm{PC}_{71} \mathrm{BM}$ as acceptor, while a PBDTTPD polymer with a combination of branched and linear substituents yields a doubling of the PCE to $8 \%$. By using transient optical spectroscopy it is shown that while the exciton dissociation and ultrafast charge generation steps are not strongly affected by the side chain modifications, the polymer with branched side chains exhibits a decreased rate of nongeminate recombination and a lower fraction of sub-ns geminate recombination. In turn the yield of long-lived charge carriers increases, resulting in a 33\% increase in short circuit current (Jsc). In parallel, the two polymers show distinct grazing incidence X-ray scattering (GIXS) spectra indicative of the presence of stacks with different orientation patterns in optimized thin-film BHJ devices. Independent of the packing pattern the spectroscopic data 


\section{WILEY-VCH}

also reveals the existence of polymer aggregates in the pristine polymer films as well as in both blends which trap excitons and hinder their dissociation.

\section{Introduction}

Organic photovoltaics (OPV) are considered a promising technology for sustainable solar energy conversion, because of the potential to fabricate organic solar cells using low-cost solution processing. The solution processability and modular synthesis of organic materials introduces opportunities in flexible and wearable power generation, and in photovoltaic coatings with tunable color and transparency for window-type applications. In the past recent years, new materials and material combinations have led to efficiencies of $>8 \%$ in single junction cells and over $10 \%$ in tandem architecture devices. ${ }^{[1,2]}$ In order to reach the performance and stability required for commercial applications, a fuller understanding of the material property-structure-function relationships to device performance is essential. Many performance parameters in organic semiconductor devices depend upon the morphology and packing of organic molecules/polymers within the active layer, and there are several approaches to morphology control, applicable at the synthetic stage or during the device processing steps. ${ }^{[3]}$ The side chains attached to the conjugated main-chain (to solubilize it in common organic solvents) play a critical role in determining the morphology and thereby device performance. ${ }^{[4]}$ Recently, bulk heterojunction solar cells based on a class of donor-acceptor copolymers containing alternating benzo[1,2-b:4,5-b']-dithiophene (BDT) and thieno[3,4-c]pyrrole-4,6-dione (TPD) units have demonstrated power conversion efficiencies $(\mathrm{PCE})>8 \%$, and open circuit voltages $\left(\mathrm{V}_{\mathrm{oc}}\right)$ of $1 \mathrm{~V} \cdot{ }^{[2 b]}$ In PBDTTPD polymer donors, the nature of the side chain pattern appears to strongly influence device performance. Thus, in recent work, ${ }^{[2 b]}$ we demonstrated that the replacement of the linear alkyl chain on the BDT unit with a branched ethylhexyl substituent leads to a near doubling in PCE in optimized bulk-heterojunction (BHJ) solar cells with phenyl-C61/71-butyric acid methyl ester (PCBM) 


\section{WILEY-VCH}

as the acceptor, by improving both the short circuit current $\left(\mathrm{J}_{\mathrm{sc}}\right)$ and fill factor $(\mathrm{FF})$. GIXS analyses show that the linear and branched patterns can lead to different preferential orientations of the polymer aggregates in the BHJ solar cell, with the branched side chains leading to preferential 'face-on' orientations (with respect to the substrate). A number of independent reports have suggested that preferential aggregate orientations can be a contributor to improved vertical charge transport, ${ }^{[3 f]}$ and charge extraction (via higher $\mathrm{J}_{\mathrm{sc}}$ and FF).

In this work, we examine the impact of the side-chain pattern of PBDTTPD on charge generation, separation, and recombination, using broadband transient absorption spectroscopy across ten decades of time resolution and a wide (visible-NIR) spectral range. We find that side chains do not significantly affect the conversion of polymer excitons into free charges but do strongly influence both the rate of non-geminate recombination and the extent of fast geminate recombination. The suppression of non-geminate recombination leads to the improvement in FF, while the suppression of both types of recombination explains the increase in Jsc and EQE. Another important aspect of these polymer blends revealed by our transient spectroscopy analyses is the presence of intermolecular aggregate states that act as exciton traps. Using a combination of photoluminescence and transient absorption spectroscopy, and applying a recently introduced soft-modeling data analysis technique, ${ }^{[5]}$ we find that excitons in the aggregate regions do not reach the interface with PCBM and, in turn, do not generate charges; overcoming this structural limitation could lead to an additional improvement in the already impressive power conversion efficiencies obtained using PBDTTPD polymers in BHJ solar cells with PCBM acceptors. 


\section{Results and Discussion}

\subsection{Materials and Device Performance}

a)
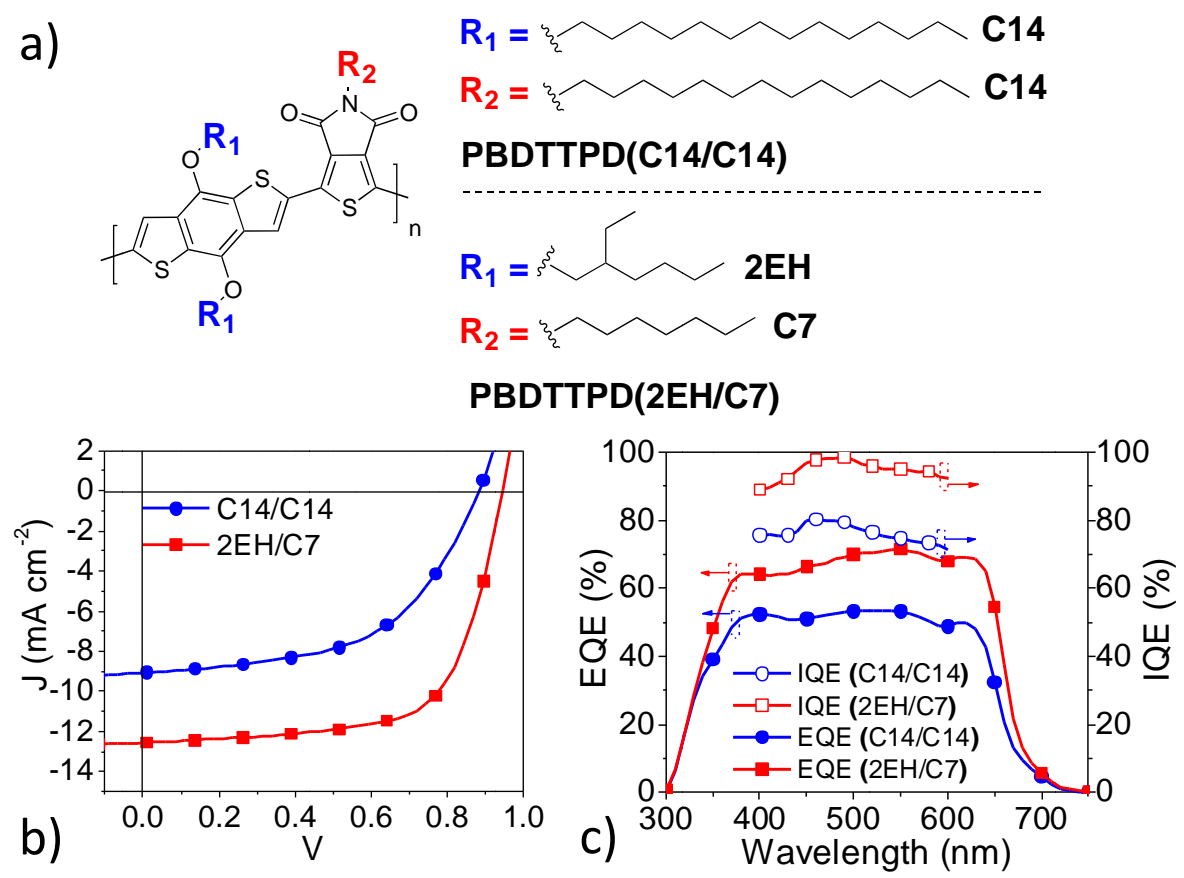

Figure 1. a) Chemical structures of PBDTTPD(2EH/C7) and PBDTTPD(C14/C14), b) representative J-V curves and c) EQE and IQE spectra for optimized PBDTTPD:PC ${ }_{71} B M$ devices. Absorption spectra are given in the Supplementary Information.

Figure 1 shows the molecular structures of the PBDTTPD analogues studied in this report, as well as the representative current density-voltage $(\mathrm{J}-\mathrm{V})$ curves, external quantum efficiency (EQE) and internal quantum efficiency (IQE) spectra of optimized BHJ solar cell devices with the acceptor $\mathrm{PC}_{71} \mathrm{BM}$. We investigated PBDTTPD polymer donors with either a combination of 2-ethyhexyl-substituted BDT and $n$-heptyl-substituted TPD motifs (PBDTTPD(2EH/C7)) or with the same linear quatrodecyl side chains on both BDT and TPD motifs (PBDTTPD(C14/C14)). Figure $1 \mathrm{~b}$ shows the J-V characteristics of solar cells optimized for the two polymers (in a 1:1.5 (wt/wt) donor:acceptor ratio with $\mathrm{PC}_{71} \mathrm{BM}$ ). As shown in Table 1, solar cells prepared from $\mathrm{PBDTTPD}(2 \mathrm{EH} / \mathrm{C} 7)$ using an optimized solvent mixture of $95 \%$ chlorobenzene (CB), 5\% chloronaphthalene (CN) exhibited an average PCE of 7.9\%, while the PBDTTPD $(\mathrm{C} 14 / \mathrm{C} 14)$ device yielded only $4.3 \%$ PCE. All the figures of merit improve in PBDTTPD(2EH/C7)-based solar cells, and the use of the processing additive $\mathrm{CN}$ during 


\section{WILEY-VCH}

device preparation allows for further improvements (Table 1). In the following spectroscopic studies we investigate the optimized blends, namely those prepared using the $\mathrm{CB} / \mathrm{CN}$ solvent mixture.

Table 1. PV Performance of PBDTTPD derivatives in standard $\mathrm{BHJ}$ devices with $\mathrm{PC}_{71} \mathrm{BM}$ (variance calculated over 10 devices), Optimized devices were prepared with a polymer:PC $\mathrm{Pl}_{71} \mathrm{BM}$ ratio of 1:1.5 (wt/wt). All devices were solution-cast from chlorobenzene (CB) containing 5\% (v/v) of the processing additive 1-chloronaphthalene (CN)

\begin{tabular}{ccccccc}
\hline $\begin{array}{c}\text { PBDTTPD } \\
\left(\mathrm{R}_{1} / \mathrm{R}_{2}\right)\end{array}$ & $\begin{array}{c}\mathrm{J}_{\mathrm{sc}} \\
{\left[\mathrm{mAcm}^{-2}\right]}\end{array}$ & $\begin{array}{c}\mathrm{V}_{\mathrm{oc}} \\
{[\mathrm{V}]}\end{array}$ & $\mathrm{FF}$ & $\begin{array}{c}\text { Avg. PCE } \\
{[\%]}\end{array}$ & $\begin{array}{c}\text { Max. PCE } \\
{[\%]}\end{array}$ & $\begin{array}{c}\text { IQE } \\
\text { @530nm } \\
{[\%]}\end{array}$ \\
\hline 2EH/C7 & $12.6 \pm 0.3$ & $0.94 \pm 0.01$ & $0.67 \pm 0.02$ & $7.9 \pm 0.2$ & 8.1 & 96 \\
$\mathrm{C} 14 / \mathrm{C} 14$ & $9.1 \pm 0.4$ & $0.88 \pm 0.02$ & $0.54 \pm 0.03$ & $4.3 \pm 0.3$ & 4.6 & 76 \\
\hline
\end{tabular}

\subsection{Spectroscopy of PBDTTPD polymers and blends}

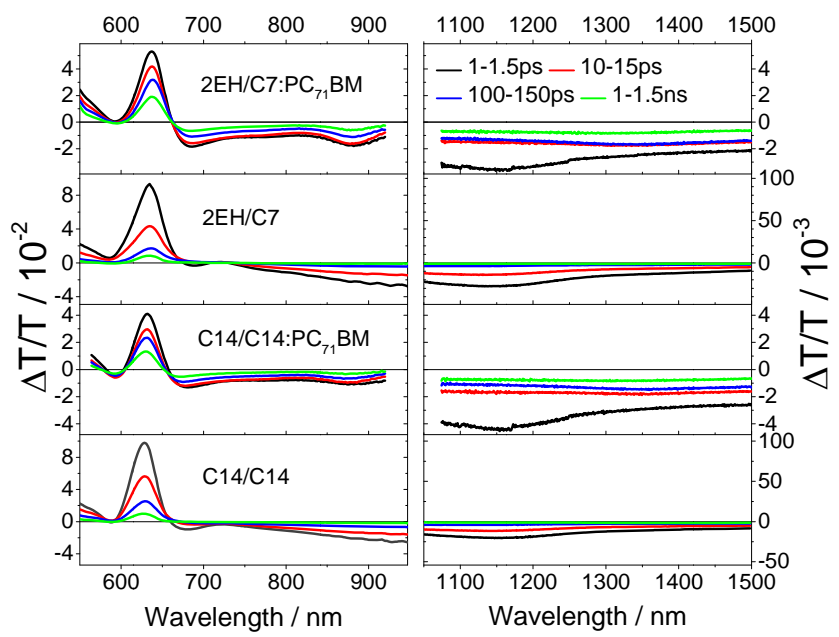

Figure 2. Transient absorption spectra integrated from 1-1.5ps (black line), 10-15ps (red line), 100-150ps (blue line) and 1-1.5ns (green line) for PBDTTPD pristine films and blends with $\mathrm{PC}_{71} \mathrm{BM}$, with the y-axis for data in the 1000-1500nm range expanded for clarity. The spectra are normalized to the fraction of photons absorbed at the excitation wavelength.

Figure 2 shows transient absorption spectra integrated over selected time intervals from 1-

$1.5 \mathrm{ps}$ to $1-1.5 \mathrm{~ns}$ for the pristine materials and blends with $\mathrm{PC}_{71} \mathrm{BM}$, exciting at $510 \mathrm{~nm}$. In the pristine films a strong positive change in transmission characteristic of bleaching of the polymer ground state is overlaid with stimulated emission (SE). Stimulated emission is also seen in the region from $700-730 \mathrm{~nm}$, consistent with emission from an intermolecular 


\section{WILEY-VCH}

aggregate state (vide infra). The bleach and stimulated emission features are overlaid on the broad photoinduced absorption (PA) of PBDTTPD excitons, extending into the near-IR (NIR) spectral region and peaking at $1150 \mathrm{~nm}$. Transient spectra of the pristine polymers on a 1-450 ns timescale are shown in the SI, and exhibit PA bands in the NIR, largely from the polymer singlet exciton. The polymer triplet is clearly seen in the quasi-steady state photoinduced absorption of the pristine polymers but not in the blends (SI), with a peak at around $1450 \mathrm{~nm}$, slightly red shifted compared to the aggregate state. It is worth noting that the polymers exhibit a ground state bleach which is red shifted by around $15 \mathrm{~nm}$ relative to the ground state absorption, and contains features which we assign to the effect of sample heating. ${ }^{[6]}$ In the blend, both the SE and exciton PA are suppressed due to dissociation of polymer excitons by ultrafast charge transfer to PCBM. A small amount of residual SE is detectable as a rise in the PA (corresponding to a decay of a positive change in transmission) in the 700$800 \mathrm{~nm}$ spectral range. In the $550-1000 \mathrm{~nm}$ wavelength range, PA signatures corresponding to charges are observed. The hole spectrum of the polymer is broad but structured, with a peak around $850-900 \mathrm{~nm}$ and resembles the charge signal seen in solution based oxidation measurements, ${ }^{[7]}$ as well as matching the long-lived PA component identified in a recent spectroscopic study. ${ }^{[8]}$ This PA is absent in the pristine films, which exhibit only the blue edge of the exciton PA in this spectra region. In all blends, charges are generated within the response time of the measurement ( $\sim 200 \mathrm{fs}$ ), with an additional small rise (time constant $\sim 2 \mathrm{ps}$ ), in agreement with the diffusion and dissociation of polymer excitons, measured in the near infrared (data and fits shown in the supporting information). This rise is suppressed under high excitation fluence $\left(>20 \mu \mathrm{Jcm}^{-2}\right)$, where the high excited state density allows bimolecular annihilation and/or recombination processes to occur at very early times. 


\section{WILEY-VCH}

\subsection{Charge generation and recombination}

We now turn to the examination of the charge generation and recombination processes in the PBDTTPD films, in order to identify the origin of the large difference in BHJ solar cell performance between the two PBDTTPD polymers.
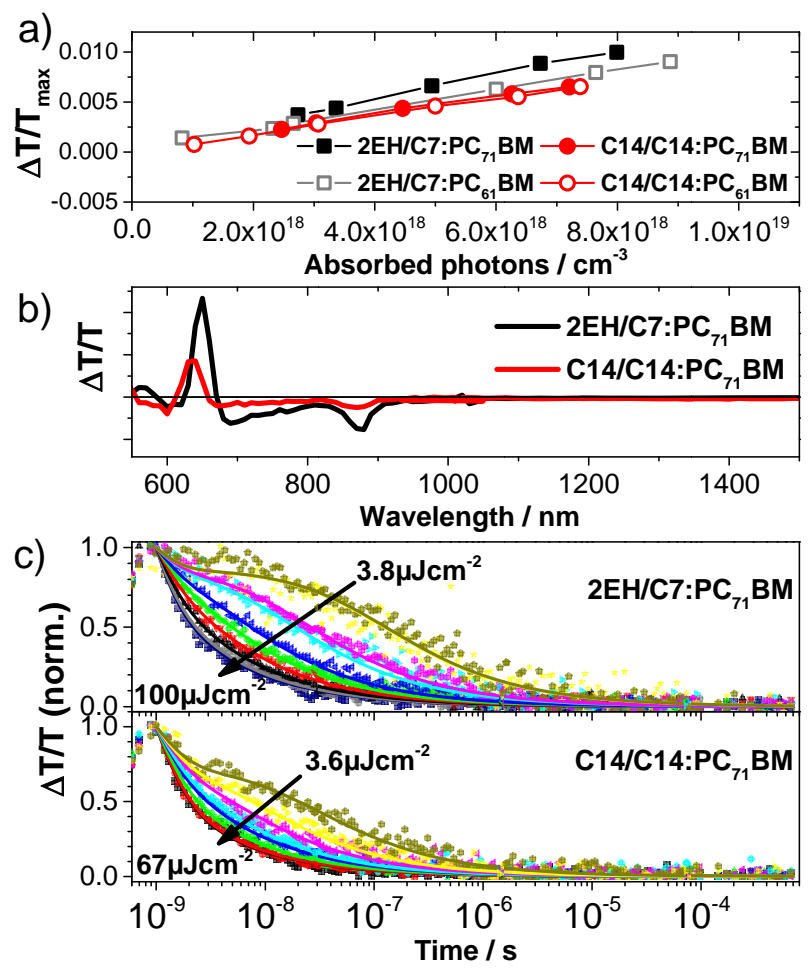

Figure 3. a) Maximum charge-induced absorption signal height integrated from $850-900 \mathrm{~nm}$ as a function of the absorbed photon density for PBDTTPD blends with either $\mathrm{PC}_{71} \mathrm{BM}$ (closed symbols) or $\mathrm{PC}_{61} \mathrm{BM}$ (open symbols) excited at 510nm. Note that the initial charge yield is similar for both polymers. b) Quasi steady-state photoinduced absorption spectra of both blends. c) Integrated decay dynamics $(850-900 \mathrm{~nm})$ on ns-ms timescale for PBDTTPD(2EH/C7):PC ${ }_{71} \mathrm{BM}$ (upper) and PBDTTPD(C14/C14):PC ${ }_{71} \mathrm{BM}$ (lower panel) blends. Solid lines are fits to the model described by Equation 1, with fit parameters given in Table 1.

Figure 3a compares the absolute PA signal height (integrated from 850-900nm, away from the region where polymer SE overlaps) as a function of the absorbed photon density, for blends of PBDTTPD(2EH/C7) and PBDTTPD(C14/C14) with $\mathrm{PC}_{71} \mathrm{BM}$ and $\mathrm{PC}_{61} \mathrm{BM}$, exciting at 510nm. The total PA signal in blends with $\mathrm{PC}_{61} \mathrm{BM}$ (where the contribution of fullerene absorption to the total charge yield is small), is independent of the polymer side chains, with $\operatorname{PBDTTPD}(2 \mathrm{EH} / \mathrm{C} 7)$ exhibiting at most an improvement in charge generation of ca. $10 \%$. 


\section{WILEY-VCH}

Since the total cross section of this state is the same in both polymers (indicated by the similarity in the plot slopes), it can be inferred that the efficiency of the initial exciton dissociation step is similar for the two polymers. When $\mathrm{PC}_{71} \mathrm{BM}$ is used as the electron acceptor, the signal in the PBDTTPD $(2 \mathrm{EH} / \mathrm{C} 7)$ blend is slightly enhanced, which may indicate slightly more favorable conditions for hole transfer; however, this is beyond the scope of the present manuscript.

A different picture emerges when monitoring the concentration and lifetime-averaged chargeinduced absorption signal of long-lived charges, which we did by quasi-steady state photoinduced absorption spectroscopy (PIA, Figure 3b). In fact, this measurement shows the time-integrated photoinduced absorption in the blend and thus represents a convolution of the population and lifetime of the excited states, namely the long-lived charges. The total PIA of long-lived charges, obtained by integrating from $700-950 \mathrm{~nm}$, is almost a factor of three larger (x2.9) in the PBDTTPD(2EH/C7) blend with $\mathrm{PC}_{71} \mathrm{BM}$ compared to $\operatorname{PBDTTPD}(\mathrm{C} 14 / \mathrm{C} 14)$, as shown in Figure 3. For blends with $\mathrm{PC}_{61} \mathrm{BM}$, the difference is slightly smaller, that is $\times 2.2$, confirming that the generation of long-lived charges from the fullerene is slightly more efficient in the $\mathrm{PC}_{71} \mathrm{BM}$ blend, consistent with the ultrafast TA measurements (data for $\mathrm{PC}_{61} \mathrm{BM}$ blends shown in SI). As we saw in Figure 3a, this difference is markedly larger than that observed at the charge generation stage, suggesting that recombination processes also play a role in the improved performance of $\operatorname{PBDTTPD}(2 \mathrm{EH} / \mathrm{C} 7)$ solar cells, and that the charged state probed in this spectral region contains a part which decays more quickly in the PBDTTPD(C14/C14) blend. In order to study the recombination dynamics of charges we therefore applied ns-ms transient absorption spectroscopy.

It is important to have in mind the key photophysical processes occurring when a photon is absorbed within the active layer of an organic solar cell: (i) photon absorption generates an excited state, namely a Coulombically-bound exciton, on the donor (acceptor) material; and (ii) this exciton typically undergoes ultrafast charge transfer at the donor-acceptor interface 


\section{WILEY-VCH}

where an energetic driving force assists the transfer of an electron (hole) from the donor (acceptor) to the acceptor (donor), while often a small fraction of the initial exciton population also shows diffusion-limited dissociation.

Although it is not yet completely clear how the free carriers are formed,${ }^{[9]}$ the result is a spatially-separated electron-hole pair. Our understanding of this charge transfer process is that within a short time typically within several picoseconds either free charges (potentially through a very short-lived delocalized CT state intermediate) or a bound CT state at the interface is formed upon charge transfer. The splitting ratio between these two populations depends on material and morphology. ${ }^{[9 c]}$ Subsequently, free charges can non-geminately recombine or be extracted from the device, while the initial CT-state population decays monomolecularly. Non-geminate recombination is frequently termed 'bimolecular recombination'; however the reaction order for this process has in several material systems been found to be greater than 2 and in many cases approaching 3 , on account of the density dependence of charge carrier mobility within an energetically disordered system, ${ }^{[10]}$ and furthermore upon the geometric restrictions at the polymer:fullerene interface. ${ }^{[9 c]}$ Figure 3c presents the dynamics of the charge PA integrated from $850-900 \mathrm{~nm}$ in PBDTTPD:PC ${ }_{71} \mathrm{BM}$ blends at excitation intensities of $4-100 \mu \mathrm{Jcm}^{-2}$. The decays are strongly intensity dependent, on account of the non-geminate charge recombination processes operating on this timescale. We previously developed a model to describe the dynamics of charge PA measured at several excitation fluences in terms of a sum of intensity dependent non-geminate recombination and intensity independent monomolecular recombination processes (Equation 1 with scheme shown in the Supporting Information). ${ }^{\left[{ }^{[c]}\right]}$ The latter can arise either by the recombination of geminate pairs and CT states, or by quasi-monomolecular, trap-limited recombination.

The derived parameters are $k_{C T \rightarrow G S}$, the rate constant for monomolecular recombination, $\lambda$ and $\gamma$, the order and rate coefficient, respectively, of non-geminate recombination, and $f$ which 


\section{WILEY-VCH}

represents the fraction of the initial population that decays by non-geminate recombination, that is the fraction of spatially-separated charge carriers. This model was developed to fit the evolution of population densities, which were determined using the transient absorption kinetics in a wavelength region in which the two populations had identical cross sections. Here, we successfully apply this model with a minor extension in order to fit transient absorption kinetics in a wavelength region in which the cross sections of the two species are not precisely known, and neither is their ratio. In this case we are not fitting kinetics that are equivalent to population densities, but nonetheless all the parameters can be extracted as above except for $f$. In our case we cannot extract the true fraction of the initial population that is in the non-geminate pool. Instead we measure a fraction that is modified by the (unknown) ratio of the cross sections, $f^{\prime}=f^{\sigma_{A}} / \sigma_{B}$ where $\sigma_{A, B}$ are the absorption cross sections of the two species and $f^{\prime}$ is the fraction of states recombining non-geminately. Although this means that, for a given sample, the fraction of absorbed photons that create free charges vs. recombining CT states cannot be directly determined, the difference in free charge generation between samples processed with different conditions can still be compared. If we extract $f^{\prime}{ }_{1}$ from the data measured on the sample prepared with polymer 1 , and $f^{\prime}{ }_{2}$ from that prepared with polymer 2, then the ratio $f^{\prime}{ }_{1} / f^{\prime}{ }_{2}$ gives the fraction more (or less) free charge generation in sample 1 than in sample 2.

$$
G S(t)=N_{0}(1-f)\left(1-\exp \left(-k_{C T \rightarrow G S}\right)\right)+N_{0} f-\left(\lambda \gamma t+\left(f N_{0}\right)^{-\lambda}\right)^{-1 / \lambda}
$$

Fits to the datasets are shown in Figure 3 and the fit parameters in

Table 2. From the non-geminate recombination coefficient and the reaction order we calculated the effective bimolecular recombination coefficient at 1 sun intensity, ${ }^{[9]}$ which is around four times larger in the PBDTTPD(C14/C14) blend. In the PBDTTPD $(2 \mathrm{EH} / \mathrm{C} 7)$ blend 


\section{WILEY-VCH}

this effective bimolecular recombination rate under 1 sun is comparable to that obtained in regioregular $\mathrm{P} 3 \mathrm{HT}: \mathrm{PC}_{61} \mathrm{BM}$ solar cells. ${ }^{[9 \mathrm{c}]}$

We also find that a sub-ns intensity independent component is required to fit the dynamics, corresponding to monomolecular (geminate) recombination of tightly bound states which experience a strong Coulombic attraction. This component represents a higher fraction of the total excited state PA (and therefore, to a higher fraction of the excited state population) in the PBDTTPD(C14/C14): $\mathrm{PC}_{71} \mathrm{BM}$ blend (26\% vs. $\left.16 \%\right)$. This in turn implies that the generation of free charges is lower in this blend, accounting, along with the faster non-geminate recombination, for the reduced $\mathrm{J}_{\mathrm{sc}}$ and lower IQE at 530nm (shown in Table 1). We now turn to an examination of the blend microstructure in order to understand the origin of the differences in recombination in blends prepared with the two PBDTTPD polymer analogues.

Table 2. Fit parameters describing charge recombination dynamics in PBDTTPD:PC ${ }_{71} \mathrm{BM}$ blends. The effective bimolecular recombination coefficient $\left(\gamma_{e f f}\right)$ at 1 sun is calculated according to reference ${ }^{[9 \mathrm{c}]}$ in order to permit comparison with other materialls systems ${ }^{[3 \mathrm{~b}, 10 \mathrm{~b}]}$.

\begin{tabular}{lll}
\hline & PBDTTPD(2EH/C7) & PBDTTPD(C14/C14) \\
& $:$ PC 71 BM & :PC 71 BM \\
\hline $\mathrm{f}$ & $0.86 \pm 0.01$ & $0.74 \pm 0.01$ \\
$\mathrm{k} / \mathrm{s}^{-1}$ & $(1.9 \pm 0.3) \times 10^{9}$ & $(1.5 \pm 0.1) \times 10^{9}$ \\
\hline$\lambda$ & $1.78 \pm 0.01$ & $1.66 \pm 0.01$ \\
\hline$\gamma /\left(\mathrm{cm}^{3}\right)^{\lambda} \mathrm{s}^{-1}$ & $(3.0 \pm 0.8) \times 10^{-25}$ & $(1.28 \pm 0.4) \times 10^{-22}$ \\
\hline$\gamma_{\mathrm{eff}} / \mathrm{cm}^{3} \mathrm{~s}^{-1}$ & $(5.2 \pm 1.4) \times 10^{-13}$ & $(2.9 \pm 1) \times 10^{-12}$ \\
\hline
\end{tabular}

\subsection{Investigation of blend morphology using TEM and GIXS}

Transmission electron microscopy (TEM, Figure 4) shows only minor differences between the phase segregation in the blends. The contrast images show that the PBDTTPD(2EH/C7):PC ${ }_{71} \mathrm{BM}$ blend exhibits fiber-like structures a few nm across, which are less well defined in the PBDTTPD $(\mathrm{C} 14 / \mathrm{C} 14)$ blend, but $\mathrm{C}$ and S atomic maps (SI) show very little difference in phase segregation between the polymers and $\mathrm{PC}_{71} \mathrm{BM}$. This is consistent with the near-identical exciton dissociation dynamics and similar charge generation yields in the two polymer:fullerene films. We investigated the microstructure in the PBDTTPD:PC ${ }_{71} \mathrm{BM}$ blends using grazing incidence $\mathrm{x}$-ray scattering (GIXS), which is 


\section{WILEY-VCH}

sensitive to both the film crystallinity and orientation of crystalline domains with respect to the substrate. GIXS patterns obtained from PBDTTPD:PC ${ }_{71} \mathrm{BM}$ blends are shown in Figure 4 (a complete series of GIXS patterns in pristine and blend films is shown for both polymers in Figure 9 of the SI). In both polymers two scattering features are observed, one corresponding to the $\pi-\pi$ stacking at $\mathrm{q} \sim 1.5-1.7 \AA^{-1}$, and a second at smaller $\mathrm{q}$, in the range $0.2-0.3 \AA^{-1}$. The latter corresponds to the lamellar spacing which is larger in PBDTTPD(C14/C14) (31 $\AA$ vs. $21 \AA$ ) on account of the longer alkyl side chains. While the $\pi$ - $\pi$ spacing in the two polymers is comparable, the angular distribution of scattering intensity points to significant differences in the ordering within polymer-rich domains in the two blend films. PBDTTPD(2EH/C7) shows a pronounced scattering intensity peak in the out-of-plane direction (i.e. along $\mathrm{q}_{\mathrm{z}}$ ), indicating that the polymer preferentially adopts a 'face-on' alignment with respect to the substrate. In $\operatorname{PBDTTPD}(\mathrm{C} 14 / \mathrm{C} 14)$, the $\pi-\pi$ scattering pattern shows as a relatively isotropic ring, indicating a combination of in-plane and out-of-plane -oriented crystallites. The correlation of preferential 'face-on' crystallite orientation with solar cell performance is in good agreement with previous reports. ${ }^{[2 b, 11]}$

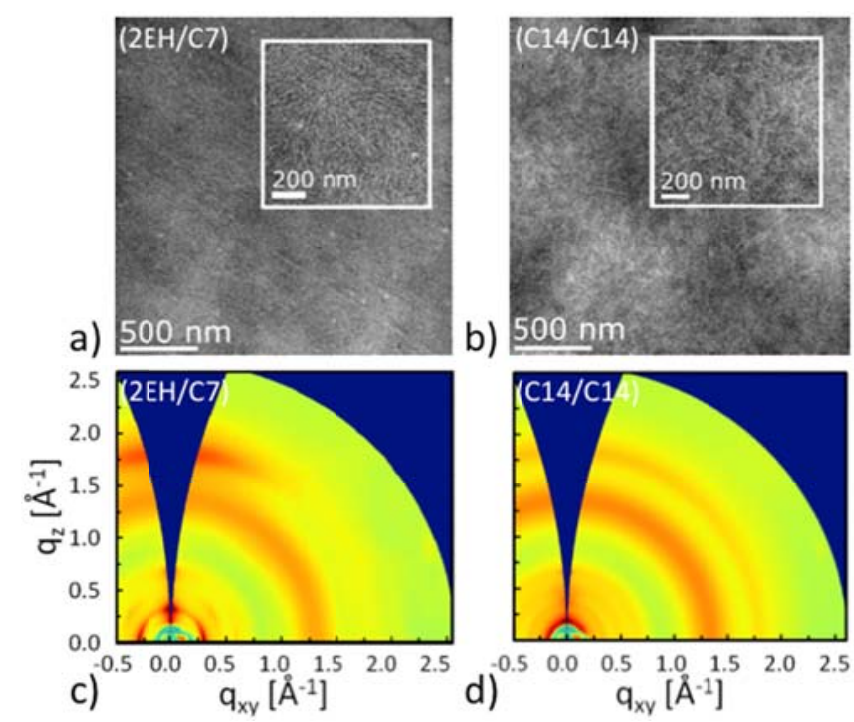

Figure 4. a,b) TEM contrast images of PBDTTPD:PC $\mathrm{P}_{71} \mathrm{BM}$ blends, showing little variation in the intermixing of donor and acceptor materials. c,d) GIXS patterns for PBDTTPD:PC ${ }_{71} B M$ blends on PEDOT-PSS substrate. PBDTTPD(2EH/C7) exhibits higher crystallinity in the $\pi-\pi$ stacking peak and a preferential face-on orientation with respect to the substrate. 


\section{WILEY-VCH}

We saw in Figure 3 how the C14/C14 substitution pattern led to an increase in the nongeminate recombination coefficient and an increase in the amount of fast geminate recombination, with a detrimental effect on both the short circuit current and the device fill factor. Charge generation and recombination are known to be intimately related to the microstructure of bulk heterojunction blends and it is increasingly recognized that material crystallization and aggregation plays a role in boosting charge separation and inhibiting recombination. In the well-studied case of P3HT:PCBM blends, it is well known that the formation of lamellar polymer aggregates induced by thermal annealing is crucial in producing the optimum photovoltaic performance, and that a key reason for this is the suppression of geminate recombination. ${ }^{[9 \mathrm{c}]}$ More recently, other studies have also shown that fullerene crystallites drive improvements in device performance, either by increasing the electron affinity of the acceptor (and thus the driving force for charge separation at the interface $)^{[12]}$ or by increasing the delocalization of interfacial charge transfer states thereby reducing geminate recombination. ${ }^{[13]}$ The results obtained in the PBDTTPD polymers are entirely consistent with a picture in which crystallinity of at least one of the components, that is the polymer in this case, facilitates charge separation by driving charges away from the interface, as the GIXS results indicate that the higher performance polymer $\operatorname{PBDTTPD}(2 \mathrm{EH} / \mathrm{C} 7)$ is also the higher crystalline derivative. Hence, it appears that the branched sidechains of PBDTTPD have a dual function, firstly they facilitate charge separation at the interface as shown in this study and secondly the aid charge extraction by influencing the packing motif as shown in earlier studies. ${ }^{[2 b, 11]}$

\subsection{Impact of polymer aggregation on exciton dissociation}

We now turn to have a closer look at the exciton dynamics in the PBDTTPD polymers and blends to complete the picture of the photophysical processes. Figure 5 shows time-resolved photoluminescence spectra of pristine PBDTTPD films. The spectra are averaged over the 


\section{WILEY-VCH}

time intervals of $0-100 \mathrm{ps}, 500-700 \mathrm{ps}$ and $1200-1500 \mathrm{ps}$ after excitation. Interestingly, in these films the PL spectra at early and late timescales differ, with the late time spectra showing a broad, unstructured and red-shifted emission. This evolution in emission is also observed in dilute solution ( $5 \times 10^{-8} \mathrm{M}$ based on monomer molecular weight in chlorobenzene/5\% chloronaphthalene solution, data shown in SI), but is absent in very dilute solution $\left(5 \times 10^{-9} \mathrm{M}\right.$ in pure chlorobenzene based on monomer molecular weight). Here, both polymers exhibit a minimal change in their spectra over time (SI). In fact, this concentration dependence suggests that the red emission arises from aggregated polymer chains. Furthermore, the contribution of the red emission to the PL increases with solution concentration (SI), supporting the assignment to PL from aggregated polymer chains. This is consistent with recently published results by Hwang et al., ${ }^{[14]}$ who showed the limited spectral evolution in a solution of PBDTTPD(2EH/C7) which had been filtered, presumably removing most or all of the polymer aggregates. In the SI a schematic of the proposed emissive species is given. To extract detailed information on the characteristics of these two emissive species, we carried out analysis on the three-dimensional data surface based on matrix factorization. We assume that the total data surface represents the sum of individual data surfaces from two species whose spectra do not change with time. These species are the intramolecular exciton (assumed to be the same as the pure exciton spectrum observed in the most dilute solution), and the emissive polymer aggregate (obtained by noting that at long delay times the data matrix is rank 1 that is, the spectrum no longer changes with time). By performing a matrix division of the two known spectra (plotted as dashed lines in the upper panels of Figure 5) we obtained their dynamics, shown in the lower panels. Further details and residuals for this factorization are found in the Supporting Information.

The lifetime of the intramolecular exciton is thus revealed to be similar in pristine PBDTTPD(2EH/C7) and PBDTTPD(C14/C14) (255 or 275 ps, respectively); while emission from the aggregated polymer decays with a time constant of 1 or $1.2 \mathrm{~ns}$, these average decay 


\section{WILEY-VCH}

lifetimes calculated from a double exponential fit to the data using the expression $\tau_{a v}=$ $\left(\alpha_{1} \tau_{1}^{2}+\alpha_{2} \tau_{2}^{2}\right) /\left(\alpha_{1} \tau_{1}+\alpha_{2} \tau_{2}\right)$, where $\alpha_{\mathrm{n}}$ and $\tau_{\mathrm{n}}$ are the amplitudes and lifetimes, respectively, of

the components of the fit. Furthermore, we can extract the rate at which the aggregated polymer emission rises, which reveals the rate of energy transfer/trapping from the intramolecular exciton into the aggregate state. This energy transfer rate does not change much between the samples, with a single exponential time constant of $55 \mathrm{ps}$ (PBDTTPD(2EH/C7)) or 40 ps (PBDTTPD(C14/C14)). All fitting was performed with nonnegative least squares in Origin 8.5 (Origin Inc.).

a)

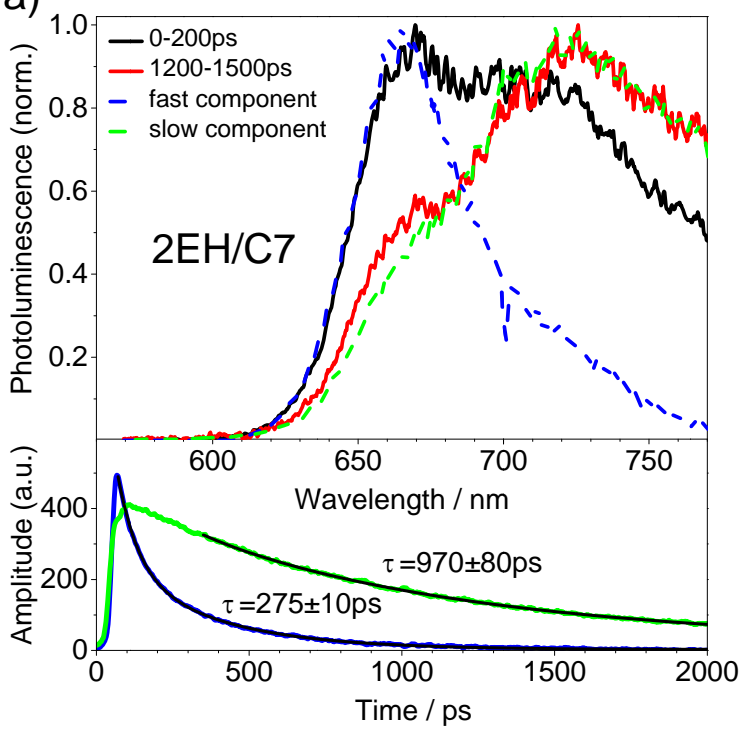

b)

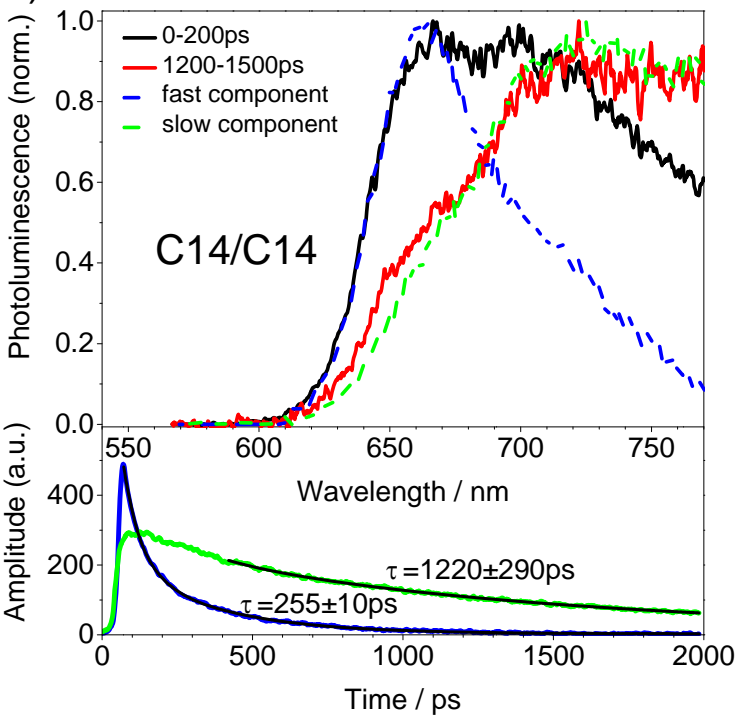

Figure 5. a) Normalized photoluminescence spectra of pristine PBDTTPD(2EH/C7) and b) PBDTTPD $(\mathrm{C} 14 / \mathrm{C} 14)$ polymer films showing the presence of two distinct features at long and short times, respectively. The spectra contain two components, the short-lived intramolecular exciton and the longer lived emission peaking at $700-750 \mathrm{~nm}$ that arises from aggregate states. Bottom panels show the dynamics of the short and long-lived components.

In the blend films with $\mathrm{PC}_{71} \mathrm{BM}$ (data shown in the Supporting Information) exciton fluorescence is efficiently quenched within the instrument response of our PL setup. However, a residual tail in the red part of the spectrum persists which resembles the spectrum of the aggregated polymer. The low intensity of this signal in relation to the background level 


\section{WILEY-VCH}

however prevents the identification of a robust late-time spectrum which could be used to perform the decomposition on this data. We therefore turned to ultrafast transient absorption spectroscopy to better understand the exciton dissocation kinetics in the blend film.

We saw earlier that in the near-infrared, PBDTTPD polymers exhibit PA bands arising from singlet excitons, which are dissociated upon blending with PCBM. Examining the spectra in detail however, reveals a spectral evolution on a ps-ns timescale which mirrors that seen in the polymer photoluminescence described above.

In order to analyze the data obtained from transient absorption, we cannot use a simple matrix factorization method as used to understand the transient photoluminescence, because we do not know both individual component spectra. Due to the high optical density required for sufficient sample absorption in a transient absorption measurement, measurement of a dilute solution is not possible and so a TA spectrum of exclusively the intramolecular exciton state cannot be measured. We may nevertheless extract the spectrum of the long-lived component by finding the time $t_{1}$ after which the data matrix is rank 1 as outlined recently by Howard et al. ${ }^{[5]}$ In the case of the pristine films, $\mathrm{t}_{1} \sim 500 \mathrm{ps}$, and the resulting spectrum after this time is shown in Figure 6 , along with the spectrum at $\mathrm{t}=1 \mathrm{ps}$ for the same samples.
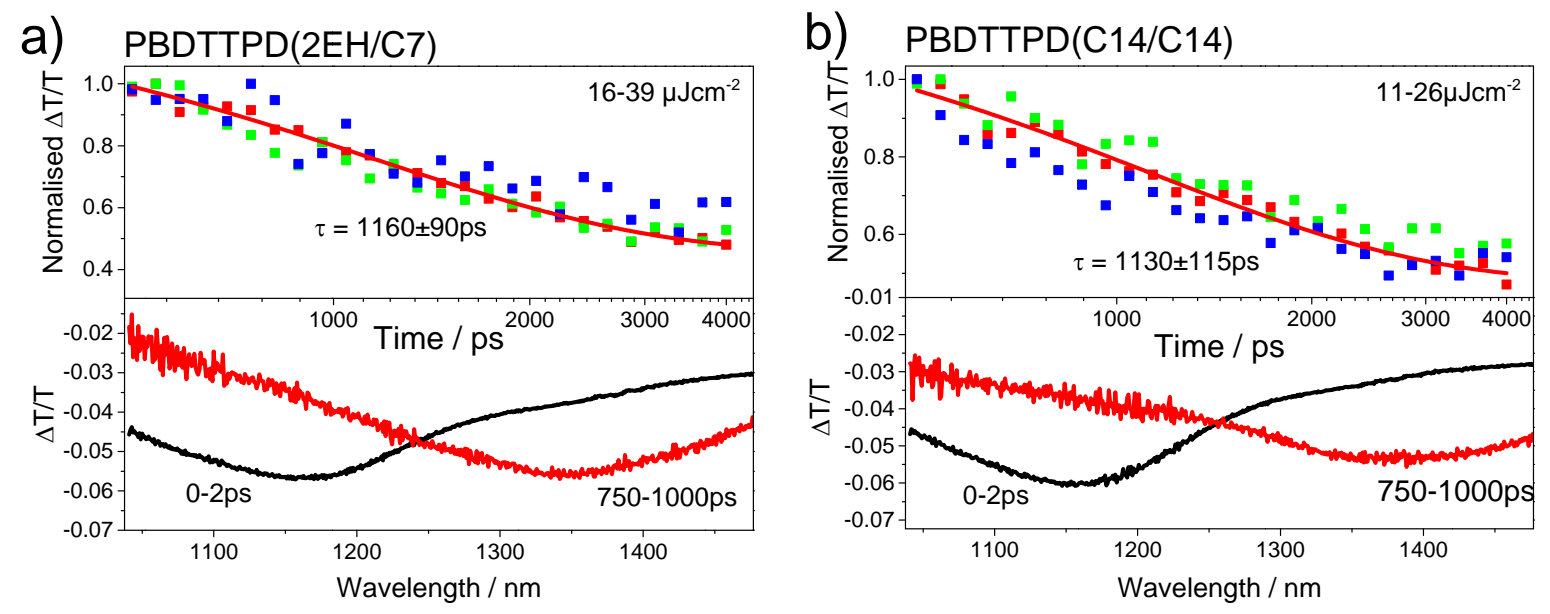


\section{WILEY-VCH}
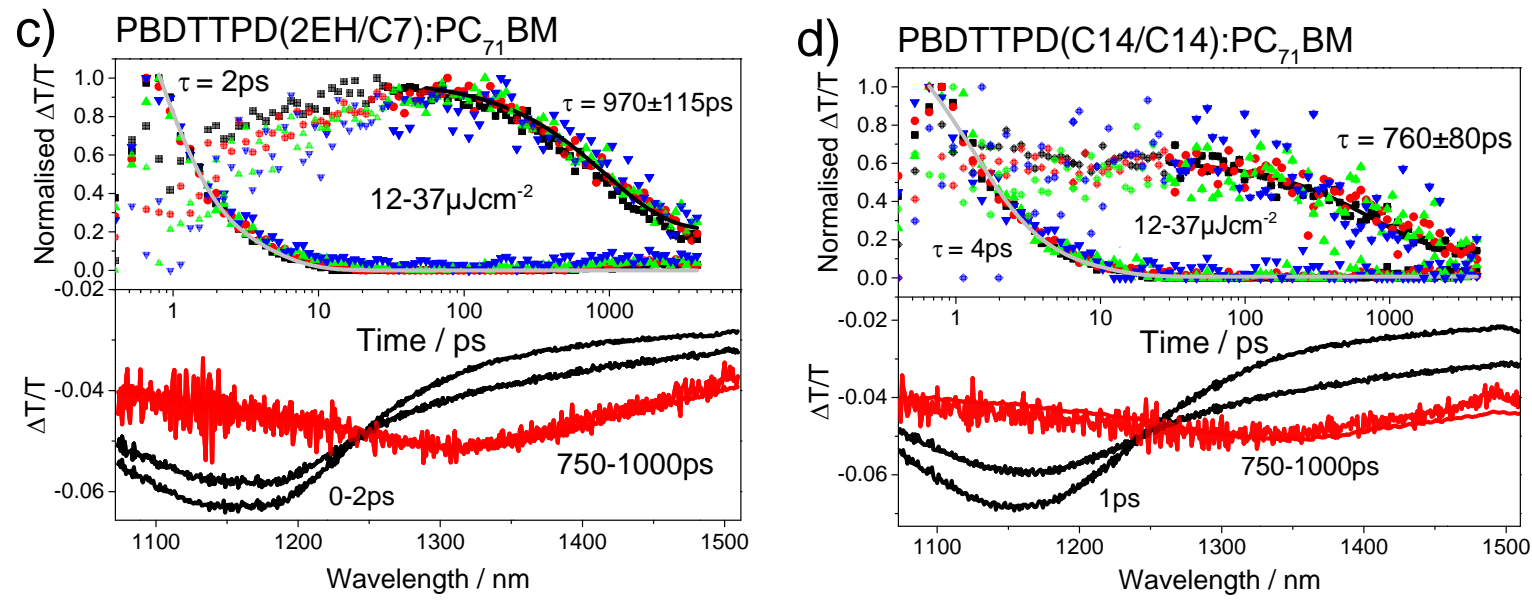

Figure 6. Near-infrared transient absorption spectra of a),b) pristine PBDTTPD films (630nm excitation) and c),d) PBDTTPD:PC ${ }_{71} \mathrm{BM}$ blends (510nm excitation). In pristine films an exponential fit to the decay after around 500ps reveals an exciton lifetime which is in good agreement with that of the aggregate state, measured using transient photoluminescence. In the blend films, the data is analysed by MCR-ALS yielding two components. The second component has the same spectral shape as the pristine aggregate, and its lifietime is similar to the lifetime obtained for the pristine film, demonstrating poor dissociation of the aggregate state.

We fit the dynamics of this species from $t_{1}$ onwards to a single exponential fit obtaining a

lifetime of around $1.1 \mathrm{~ns}$. These lifetimes are rather short compared to the $100 \mu \mathrm{s}$ measured for the PBDTTPD triplet state, ${ }^{[7]}$ but are in excellent agreement with the fluorescence lifetime of the aggregated polymer, recorded in the pristine sample, leading us to assign it to that state.

In the case of the blend films, the fast dissociation of the intramolecular exciton results in a data matrix that is rank 1 after only 50ps. By fitting the decay after $t_{1}$, as in the pristine films, we readily extract the lifetime of this PA, in the same way as for the pristine film. Here, the decay lifetimes are 970ps and 760ps for PBDTTPD(2EH/C7) and PBDTTPD(C14/C14) respectively. Due to its spectral position and nanosecond lifetime, we conclude that this PA arises from the same species in both pristine and blend films, and that it is the aggregate state. However, we note that the extracted lifetimes of the state in the blends are not strongly reduced relative to the pristine polymers. This shows that the excited states generated within aggregated polymer regions are not efficiently dissociated at the interface with $\mathrm{PC}_{71} \mathrm{BM}$ in the 


\section{WILEY-VCH}

blend films, and therefore that excitons generated in these aggregated polymer regions are unable to contribute to charge generation. This may be due to a reduced energy offset at the interface, to the fact that excitons in the low energy aggregate become trapped in regions of the polymer from which they are unable to diffuse to the donor:acceptor interface, or may indicate that these excitons have a reduced spatial extent, a parameter that recent reports have highlighted as playing a role in interfacial charge separation. ${ }^{[15]}$

We further applied a recent data analysis method to the TA data surface in order to understand the dynamics of the intramolecular polymer exciton. This soft-modelling approach is based on the multivariate curve resolution-alternating least squares (MCR-ALS) approach developed by Tauler and coworkers and freely available for implementation in MATLAB ${ }^{[16]}$ Full details of the analysis method can be found in [5] and in the Supporting Information. We applied MCR-ALS analysis to the transient absorption dynamics recorded in the $1000-1500 \mathrm{~nm}$ spectral region for both pristine and blend films. By fixing the known spectrum of the longlived species, we obtain the kinetics and spectral profiles shown in Figure 6. We thereby demonstrate that the intramolecular polymer exciton decays independently of excitation intensity with a time constant (average lifetime, double exponential fit) of $4.5 \mathrm{ps}$ in both blends. This analysis method allows us to track the exciton dynamics in PBDTTPD:PCBM blends, beyond the temporal evolution of our Streak Camera setup, where the measurement of fluorescence quenching is limited by the ca. 10ps instrument response.

\section{Conclusion}

In conclusion, we have used time resolved photoluminescence and transient absorption spectroscopy to characterize charge generation and recombination dynamics in PBDTTPD:PCBM blends, and to investigate the origin of the improvement in performance achieved by subtle changes in the polymer molecular structure. A branched-linear side chain substitution pattern leads to a doubling of power conversion efficiency through improvements 


\section{WILEY-VCH}

in all figures of merit, compared to a polymer containing only linear side chains. We have shown that the initial charge transfer yield is not sufficiently affected by the side chain substitution pattern to explain the large difference in the long-lived charge PA; exciton dissociation (as measured by the fluorescence quenching) is efficient in both blends and the charge yields comparable on an early timescale. Instead we find that in the branched/linear PBDTTPD $(2 \mathrm{EH} / \mathrm{C} 7)$ polymer both the fraction of geminate recombination and the rate of non-geminate recombination are lower, and we correlate these changes to the extent of formation of ordered and crystalline polymer domains within this blend.

In films and in solutions of concentrations above $5 \times 10^{-9} \mathrm{M}$, PBDTTPD polymers furthermore exhibit emission from an aggregate state, as well as from the pure intramolecular singlet state. We were able to characterise the lifetimes of the intramolecular (220ps) and the intermolecular (1ns) excited states in thin films. The ability of time-resolved photoluminescence to characterise the exciton dissociation in PBDTTPD blends is limited by the instrument response of the measurement and furthermore by the high background level in the range of polymer aggregate emission within our setup, however by combining these measurements with picosecond to nanosecond transient absorption spectroscopy, we were able to characterize the intramolecular and intermolecular states on an ultrafast timescale. Our analyses reveal that while the intramolecular polymer exciton in PBDTTPD is dissociated highly efficiently (with an inverse rate of $4.5 \mathrm{ps}$ ), dissociation of excited states located on the aggregated polymer in the blend is poor. However, losses through this pathway are small, as indicated by the high measured IQE, especially in the case of PBDTTPD(2EH/C7).

The effects of faster non-geminate, and increased geminate, recombination contribute to the lower short circuit current and fill factor in PBDTTPD(C14/C14) solar cells. Furthermore, an interesting aspect of the photophysics of these polymers is the inefficient dissociation of intermolecular aggregate excited states by PCBM, which can act as an additional contributing loss pathway. Taken together these results provide a detailed picture of the efficiency-limiting 


\section{WILEY-VCH}

processes in PBDTTPD solar cells. Crystalline polymer domains are required to suppress geminate and bimolecular recombination; however, the reduced dissociation of excitons in aggregated polymer regions may act as an energetic trap for excitons, which in turn cannot contribute to the BHJ device photocurrent.

\section{Experimental Section}

Methods and Materials: All reagents from commercial sources were used without further purification. Reactions were carried out under nitrogen atmosphere. Solvents were dried and purified using standard techniques. Flash chromatography was performed with analyticalgrade solvents using Silicycle Silica Flash P60 (particle size 40-63 $\mu \mathrm{m}, 60 \AA, 230-400$ mesh) silica gel. Flexible plates PE SilG/UV $250 \mu \mathrm{m}$ from Whatman ${ }^{\circledR}$ were used for TLC. Compounds were detected by UV irradiation or staining with $\mathrm{I}_{2}$, unless otherwise stated. All compounds were characterized by ${ }^{1} \mathrm{H}$ NMR $(400 \mathrm{MHz})$ and ${ }^{13} \mathrm{C}$ NMR $(100 \mathrm{MHz})$ on a Bruker Avance III Ultrashielded 400 Plus instrument and acquired at room temperature. Highresolution mass spectrometry (HRMS) data were recorded using a Thermo Scientific - LTQ Velos Orbitrap MS in positive electro spray ionization (+ESI) or positive atmospheric pressure photoionization (+APPI) mode. Elemental analyses were carried out on a Flash 2000 - Thermo Scientific CHNO Analyzer. Size exclusion chromatography (SEC) was performed with 1,3,5-trichlorobenzene (TCB) at an elution rate of at $1.0 \mathrm{~mL} / \mathrm{min}$ (injection volume: 200 $\mu \mathrm{L})$ through a PLgel MIXED-B column $(10 \mu \mathrm{m})(+\mathrm{PLgel}$ guard $)$, at $135^{\circ} \mathrm{C}$. The SEC system consisted of an Alliance 2000 separation module equipped with RI detector. The apparent molecular weights and polydispersities $\left(M_{w} / M_{n}\right)$ were determined with a calibration based on linear polystyrene (PS) standards. UV-Vis were carried out on a Varian Cary 100 (in chlorobenzene and on glass substrates). "Photoelectron Spectroscopy in Air" (PESA) measurements were recorded using a Riken Keiki Model AC-2 spectrometer with a power setting of $10 \mathrm{nW}$ and a power number of 0.33 . Samples for PESA were prepared on glass substrates. 
Synthetic Procedures:

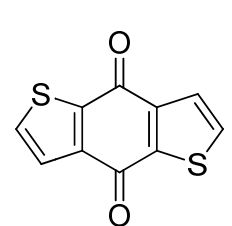

1

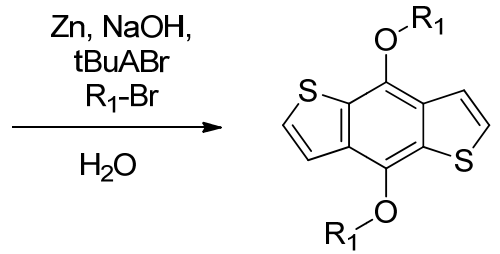

2, $\mathrm{R}_{1}=$ 2-ethylhexyl

3, $\mathrm{R}_{1}=n$-tetradecyl

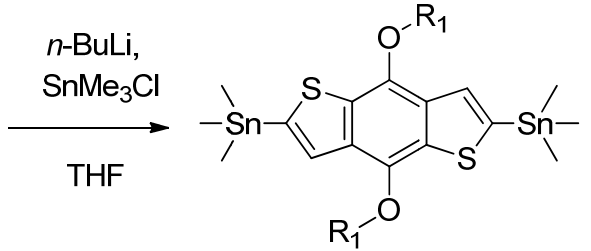

4, $\mathrm{R}_{1}=2$-ethylhexyl $\quad 81 \%$ 5, $\mathrm{R}_{1}=n$-tetradecyl $\quad 72 \%$

Benzo[1,2-b:4,5-b']dithiophene-4,8-dione $\quad(1)^{1} \quad$ 4,8-bis((2-ethylhexyl)oxy)benzo[1,2-b:4,5$\mathrm{b}^{\prime}$ ]dithiophene $(2)^{2}$ and 4,8-bis(tetradecyloxy)benzo[1,2-b:4,5-b']dithiophene $(3)^{3}$ were synthesized according to previously reported methods.

General procedure for the stanyllation of 4,8-bis(alkyloxy)benzo[1,2-b:4,5-b']dithiophene (BDT): Compound 2 or 3 (4 mmol) were solubilized in $75 \mathrm{~mL}$ of dry THF under inert atmosphere. The mixture was cooled down to $-78^{\circ} \mathrm{C}$ using a dry ice-acetone bath, and 4.55 $\mathrm{mL}$ of $n$-butyllithium ( $8.8 \mathrm{mmol}, 2.5 \mathrm{M}$ in $n$-hexane) was added dropwise. After being stirred at $-78^{\circ} \mathrm{C}$ for $1 \mathrm{~h}$, the solution was slowly warmed up to room temperature and stirred for 30 min. The cloudy mixture was cooled in the dry ice-acetone bath, and trimethyltin chloride $(1.99 \mathrm{~g}, 10 \mathrm{mmol})$ was added in one portion (the mixture turned clear). The reaction mixture was stirred overnight at room temperature, was then poured into $200 \mathrm{~mL}$ of cool water, and was extracted with diethyl ether $(\mathrm{x} 4)$. The organic layers were combined, washed with brine (x1), dried over anhydrous $\mathrm{MgSO}_{4}$ and concentrated under vacuum. The residue was recrystallized twice from ethanol to yield the desired compounds as colorless needles.

(4-((2-ethylhexyl)oxy)-8-((3-ethylhexyl)oxy)benzo[1,2-b:4,5-b']dithiophene-2,6

diyl)bis(trimethylstannane) (4): (2.5 g, 81\%). ${ }^{1} \mathrm{H} \mathrm{NMR}\left(\mathrm{CDCl}_{3}, 400 \mathrm{MHz}\right): \delta(\mathrm{ppm})=7.51(\mathrm{~s}$, 2H), $4.19(\mathrm{~d}, J=5.41 \mathrm{~Hz}, 4 \mathrm{H}), 1.81(\mathrm{~m}, 2 \mathrm{H}), 1.73-1.31(\mathrm{~m}, 16 \mathrm{H}), 1.02$ (t, $J=7.44 \mathrm{~Hz}, 6 \mathrm{H})$, $0.94(\mathrm{t}, J=6.77 \mathrm{~Hz}, 6 \mathrm{H}), 0.44(\mathrm{~s}, 18 \mathrm{H}) .{ }^{13} \mathrm{C} \mathrm{NMR}\left(\mathrm{CDCl}_{3}, 100 \mathrm{MHz}\right): \delta(\mathrm{ppm})=142.9$, 140.0, 133.5, 132.5, 127.6, 75.3, 40.3, 30.2, 28.9, 23.6, 22.8, 13.9, 11.0, -8.7. HRMS (+APPI, $m / z$ ): calcd. for $\mathrm{C}_{32} \mathrm{H}_{54} \mathrm{O}_{2} \mathrm{~S}_{2} \mathrm{Sn}_{2}[\mathrm{M}]^{+}:$: 774.1609; found, 774.1595.

(4,8-bis(tetradecyloxy)benzo[1,2-b:4,5-b']dithiophene-2,6-diyl)bis(trimethylstannane)

(2.7 g, 72\%). ${ }^{1} \mathrm{H} \mathrm{NMR}\left(\mathrm{CDCl}_{3}, 400 \mathrm{MHz}\right): \delta(\mathrm{ppm})=7.51(\mathrm{~s}, 2 \mathrm{H}), 4.29(\mathrm{t}, J=6.56 \mathrm{~Hz}, 4 \mathrm{H})$, 1.88 (quintuplet, $J=7.15 \mathrm{~Hz}, 4 \mathrm{H}), 1.55(\mathrm{~m}, 4 \mathrm{H}), 1.36(\mathrm{~m}, 40 \mathrm{H}), 0.87(\mathrm{t}, J=6.53 \mathrm{~Hz}, 6 \mathrm{H})$, $0.44(\mathrm{~s}, 18 \mathrm{H}) .{ }^{13} \mathrm{C} \mathrm{NMR}\left(\mathrm{CDCl}_{3}, 100 \mathrm{MHz}\right): \delta(\mathrm{ppm})=143.1,140.5,134.0,132.9,128.0,73.6$, 31.9, 30.5, 29.7, 29.6, 29.5, 29.4, 26.1, 22.7, 14.1, 8.3. HRMS (+APPI, $m / z)$ : calcd. for $\mathrm{C}_{44} \mathrm{H}_{78} \mathrm{O}_{2} \mathrm{~S}_{2} \mathrm{Sn}_{2}[\mathrm{M}]^{+}:$: 942.3487; found, 942.3482 . 


\section{WILEY-VCH}

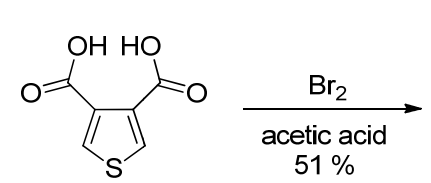

6

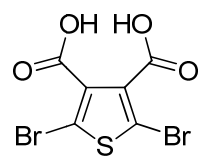

7

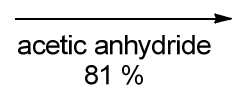

$81 \%$

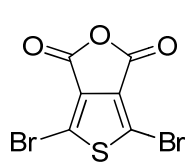

8

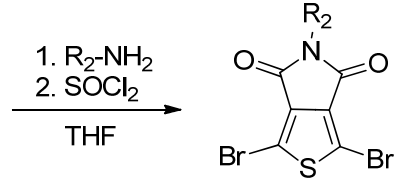

9, $\mathrm{R}_{2}=n$-heptyl

$10, \mathrm{R}_{2}=n$-tetradecyl
$86 \%$ $89 \%$

2,5-Dibromothiophene-3,4-dicarboxylic acid $(7)^{3}$ and 4,6-Dibromothieno[3,4-c]furan-1,3dione $(8)^{4}$ were synthesized according to previously reported methods.

General procedure for the preparation of 1,3-Dibromo-5-(alkyl)-4H-thieno[3,4-c]pyrrole4,6(5H)-dione (TPD). Compound 8 (1.46 g, $4.68 \mathrm{mmol})$ was combined with $12 \mathrm{~mL}$ of dry THF in a $50 \mathrm{~mL}$ flask, and alkylamine $(4.90 \mathrm{mmol})$ was added. The reaction mixture was stirred at $50^{\circ} \mathrm{C}$ for $3 \mathrm{~h}$. The volatiles were removed in vacuo, thionyl chloride $(5 \mathrm{~mL})$ was added to the residue, and the reaction mixture was stirred at $55^{\circ} \mathrm{C}$ for $4 \mathrm{~h}$. The reaction contents were carefully added dropwise to a mixture of water $(100 \mathrm{~mL})$ and methanol $(50 \mathrm{~mL})$. The precipitate was filtered, dried and purified by column chromatography using $\mathrm{CHCl}_{3}$ as the eluent. The $\mathrm{CHCl}_{3}$ solution was concentrated by evaporation, and the solid was finally recrystallized from ethanol to yield the corresponding TPD monomer as white flakes (9-10).

1,3-dibromo-5-heptyl-4H-thieno[3,4-c]pyrrole-4,6(5H)-dione (9): (1.64 g, 86\%). ${ }^{1} \mathrm{H}$ NMR $\left(\mathrm{CDCl}_{3}, 400 \mathrm{MHz}\right): \delta(\mathrm{ppm})=3.58(\mathrm{t}, J=7.28 \mathrm{~Hz}, 2 \mathrm{H}), 1.64(\mathrm{~m}, 2 \mathrm{H}), 1.30(\mathrm{~m}, 8 \mathrm{H}), 0.87(\mathrm{t}, J$ $=6.65 \mathrm{~Hz}, 3 \mathrm{H}) .{ }^{13} \mathrm{C} \mathrm{NMR}\left(\mathrm{CDCl}_{3}, 100 \mathrm{MHz}\right): \delta(\mathrm{ppm})=160.21,134.6,112.7,38.6,31.4$, 28.6, 28.1, 26.6, 22.4, 13.9. HRMS (+ESI, $m / z$ ): calcd. for $\mathrm{C}_{13} \mathrm{H}_{16} \mathrm{Br}_{2} \mathrm{NO}_{2} \mathrm{~S}[\mathrm{M}+\mathrm{H}]^{+}$: 422.9268; found, 407.9263. Anal. calcd. for $\mathrm{C}_{13} \mathrm{H}_{15} \mathrm{Br}_{2} \mathrm{NO}_{2} \mathrm{~S}$ : C, 38.16; H, 3.10; N, 3.42; $\mathrm{S}$ : 7.84; found: C, 38.33; H, 3.66; N, 3.46; S: 7.87.

1,3-dibromo-5-tetradecyl-4H-thieno[3,4-c]pyrrole-4,6(5H)-dione (10): (2.1 g, 89\%). NMR $\left(\mathrm{CDCl}_{3}, 400 \mathrm{MHz}\right): \delta(\mathrm{ppm})=3.58(\mathrm{t}, J=7.2 \mathrm{~Hz}, 2 \mathrm{H}), 1.63(\mathrm{~m}, 2 \mathrm{H}), 1.24(\mathrm{~m}, 22 \mathrm{H}), 0.87(\mathrm{t}, J$ $=6.8 \mathrm{~Hz}, 3 \mathrm{H}) .{ }^{13} \mathrm{C} \mathrm{NMR}\left(\mathrm{CDCl}_{3}, 100 \mathrm{MHz}\right): \delta(\mathrm{ppm})=160.4,134.8,112.9,38.9,31.9,29.7$, 29.6, 229.5, 29.4, 29.3, 29.1, 28.2, 26.8, 22.7, 14.1. HRMS (+ESI, $m / z)$ : calcd. for $\mathrm{C}_{20} \mathrm{H}_{30} \mathrm{Br}_{2} \mathrm{NO}_{2} \mathrm{~S}[\mathrm{M}+\mathrm{H}]^{+}:$506,0364; found, 506.0360. Anal. calcd. for $\mathrm{C}_{20} \mathrm{H}_{29} \mathrm{Br}_{2} \mathrm{NO}_{2} \mathrm{~S}$ : C, 47.35; H, 5.76; N, 2.76; S: 6.32; found: C, 47.36; H, 6.06; N, 2.76; S: 6.46.

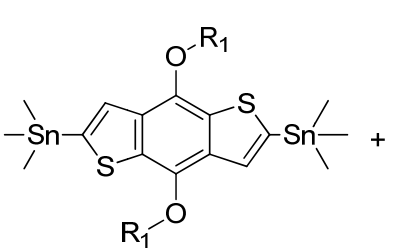

4, $\mathrm{R}_{1}=$ 2-ethylhexyl (2EH) 5, $\mathrm{R}_{1}=n$-tetradecyl (C14)

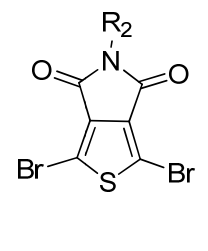

9, $\mathrm{R}_{2}=n$-heptyl (C7) 10, $\mathrm{R}_{2}=n$-tetradecyl $(\mathrm{C} 14)$
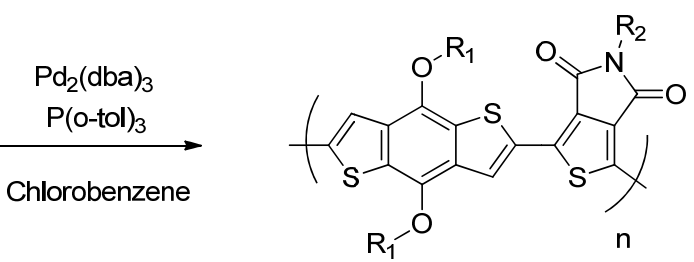

PBDTTPD(2EH/C7): $\quad \mathrm{R}_{1}=$ 2-ethylhexyl; $\mathrm{R}_{2}=n$-heptyl PBDTTPD(C14/C14): $\mathrm{R}_{1}=n$-tetradecyl; $\mathrm{R}_{2}=n$-tetradecyl
$88 \%$ $92 \%$ 


\section{WILEY-VCH}

General procedure for the polymerization: 1,3-Dibromo-5-(n-alkyl)-4H-thieno[3,4-c]pyrrole4,6(5H)-dione $\quad(516 \mu \mathrm{mol}), \quad$ (4,8-bis(alkyloxy)benzo[1,2-b:4,5-b']dithiophene-2,6diyl)bis(trimethylstannane) $(500 \mu \mathrm{mol})$, tris(dibenzylideneacetone)dipalladium (14.2 $\mathrm{mg}, 15.5$ $\mu \mathrm{mol})$ and tri-o-tolylphosphine $(18.9 \mathrm{mg}, 62.1 \mu \mathrm{mol})$ were combined in a $50 \mathrm{~mL}$ Schlenk flask. Then, $9.1 \mathrm{~mL}$ of chlorobenzene freshly degassed via F.P.T cycles were added to the flask and the reaction mixture was stirred for $36 \mathrm{~h}$ at $110^{\circ} \mathrm{C}$. Next, the polymerization mixture was cooled down to $55^{\circ} \mathrm{C}$, and the strong complexing ligand N,N-diethyl-2phenyldiazenecarbothioamide $(34 \mathrm{mg}, 155 \mu \mathrm{mol})$ was added with $\mathrm{CHCl}_{3}(20 \mathrm{~mL})$. After stirring for $3 \mathrm{~h}$ under inert atmosphere, the mixture was slowly precipitated into methanol $(200 \mathrm{~mL})$. The precipitate was filtered through a Soxhlet thimble and purified via Soxhlet extraction for $12 \mathrm{~h}$ with methanol followed by $8 \mathrm{~h}$ with hexane. The polymer was finally collected from chlorobenzene. The resulting solution was then concentrated by evaporation, precipitated into methanol $(\approx 400 \mathrm{~mL})$ and filtered to yield the desired polymers. The polymerization yields and SEC results are shown in Table S1.

Table S1. SEC analyses of the PBDTTPD derivatives

\begin{tabular}{ccccc}
\hline PBDTTPD $\left(\mathrm{R}_{1} / \mathrm{R}_{2}\right)$ & $\begin{array}{c}\text { Yield } \\
{[\mathrm{mg}]}\end{array}$ & $\begin{array}{c}M_{n} \\
{[\mathrm{kDa}]}\end{array}$ & $\begin{array}{c}M_{w} \\
{[\mathrm{kDa}]}\end{array}$ & PDI \\
\hline$(2 \mathrm{EH} / \mathrm{C} 7)$ & $\begin{array}{c}305 \\
88 \%\end{array}$ & 10.8 & 44.3 & 4.1 \\
$(\mathrm{C} 14 / \mathrm{C} 14)$ & $\begin{array}{c}440 \\
92 \%\end{array}$ & 11.5 & 33.7 & 2.9 \\
\hline
\end{tabular}

Device Fabrication: The solar cells were prepared on glass substrates with tin-doped indium oxide (ITO, $15 \Omega \mathrm{sq}^{-1}$ ) patterned on the surface. Substrates were first scrubbed with dilute Extran 300 detergent solution to remove organic residues before immersing in an ultrasonic bath of dilute Extran 300 for $15 \mathrm{~min}$. Samples were rinsed in flowing deionized water for 5 min before being sonicated for $15 \mathrm{~min}$ each in successive baths of acetone and isopropanol. After a final $5 \mathrm{~min}$ rinse in flowing deionized water, samples were dried with pressurized nitrogen before being exposed to a UV-ozone plasma for $15 \mathrm{~min}$. An aqueous solution of PEDOT:PSS (Clevios P VP AI 4083) was spin-cast at 4,000 rpm onto the substrates and baked at $140^{\circ} \mathrm{C}$ for $15 \mathrm{~min}$. All steps above were performed in a laminar flow hood to prevent adsorption of dust particles on the substrates. Immediately after baking the substrates, the samples were then transferred into a dry nitrogen glovebox $(<3 \mathrm{ppm} \mathrm{O} 2)$ for active layer deposition. 


\section{WILEY-VCH}

All solutions were prepared in the glovebox using the polymers synthesized as previously described (vide supra) and $\mathrm{PC}_{71} \mathrm{BM}$ purchased from SOLENNE. The polymers and $\mathrm{PC}_{71} \mathrm{BM}$ were dissolved in chlorobenzene containing 5\% (by volume) of 1-chloronaphthalene (CN) additive, and the solutions were stirred overnight at $115^{\circ} \mathrm{C}$. Optimized devices were prepared using a polymer: $\mathrm{PC}_{71} \mathrm{BM}$ ratio of 1:1.5 (by weight), with a concentration of $20 \mathrm{mg} \mathrm{mL}^{-1}$. The effects of various solvents, solution concentrations, additive concentrations, and blend ratios on device performance were also examined.

The active layers were spin-cast from the solutions at $90^{\circ} \mathrm{C}$ at an optimized speed for $45 \mathrm{~s}$, using a programmable spin coater from Specialty Coating Systems (Model G3P-8), resulting in a film of 100 to $120 \mathrm{~nm}$ in thickness. The samples were then dried at room temperature for 1 hour. Next, the samples were placed in a thermal evaporator for evaporation of $7 \mathrm{~nm}$ thickness calcium evaporated at $0.5 \AA \mathrm{s}^{-1}$ and $120 \mathrm{~nm}$ of aluminum electrodes evaporated at 5 $\AA \mathrm{s}^{-1}$, at a pressure less than $1 \times 10^{-6}$ Torr. Following electrode deposition, samples underwent $\mathrm{J}-\mathrm{V}$ testing.J-V measurements of solar cells were performed in the glovebox with a Keithley 2400 source meter and an Oriel Sol3A Class AAA solar simulator calibrated to 1 sun, AM1.5 G, with a KG-5 silicon reference cell certified by Newport. The external quantum efficiency (EQE) measurements were performed at zero bias by illuminating the device with monochromatic light supplied from a Xenon arc lamp in combination with a dual-grating monochromator. The number of photons incident on the sample was calculated for each wavelength by using a silicon photodiode calibrated by NIST.

Internal Quantum Efficiency (IQE): Active layer optical constants were measured with a Woollam M2000 spectroscopic ellipsometer and film thicknesses were measured with a Veeco Dektak profilometer. To calculate the IQE, the device absorption was measured with an integrating sphere to collect scattered light, and the active layer absorption was isolated by subtracting parasitic electrode absorptions, calculated by transfer matrix modeling, from the total device absorption.

Preparation of samples for spectroscopy experiments: Quartz substrates (Präzisions Glas \& Optik $\mathrm{GmbH}$ ) were cleaned by scrubbing with Hellmanex detergent solution and rinsing thoroughly with deionized water before ultrasonication in isopropanol for 10 minutes. Finally, the substrates were exposed to Ar plasma for 10 minutes. Solutions of the blend components in a 1:1.5 polymer:fullerene ratio were prepared using either a $95 \%$ chlorobenzene $/ 5 \%$ chloronaphthalene mixture, or with chloroform. Chlorobenzene/chloronaphthalene solutions 


\section{WILEY-VCH}

were stirred at $110^{\circ} \mathrm{C}$ overnight, while chloroform solutions were stirred at room temperature. The active layers were spin coated at an optimized speed (typically 1200rpm) for $45 \mathrm{~s}$. Samples were left to dry for an hour before loading into a sample holder under a dynamic vacuum of $10^{-5}$ mbar. All sample preparation and sample transfer was carried out in a $\mathrm{N}_{2}$ filled glovebox.

For solution spectroscopy measurements, the polymers were dissolved in a $95 \%$ chlorobenzene $/ 5 \%$ chloronaphthalene solvent mixture to concentrations from $1 \times 10^{-9}$ to $5 \times 10^{-}$ ${ }^{8} \mathrm{M}$ (based on monomer molecular weight).

Quasi steady state photoinduced absorption spectroscopy: Quasi steady-state photoinduced absorption spectroscopy (PIA) was performed with a home built pump-probe setup. A $100 \mathrm{~W}$ tungsten-halogen lamp with a LOT Oriel Omni- $\lambda 300$ monochromator was used as the probe and a Newport LED (LED-527-HP) working at $524 \mathrm{~nm}$ with $100 \mathrm{mWcm}^{-2}$ used as a pump. The samples were placed in a nitrogen-cooled optical cryostat (Oxford Instruments Optistat $\mathrm{CF})$ at $80 \mathrm{~K}$ in helium atmosphere. The transmitted light was dispersed by a second identical monochromator and then detected by a photodetector. For measurements in the wavelength range from 500 to $1100 \mathrm{~nm}$, an amplified silicon photodetector (Thorlabs PDA 100A) was employed, which was replaced by an amplified germanium detector (Thorlabs PDA 50B) for the wavelength range from 900 to $1800 \mathrm{~nm}$. The pump light was chopped at $317 \mathrm{~Hz}$ to induce changes in the transmission $\Delta \mathrm{T}$, which were measured by using a lock-in amplifier (EG\&G Princeton Applied Research model 5210). To calculate $\Delta \mathrm{T} / \mathrm{T}$, the transmission was recorded prior to the PIA measurement and corrected for photoluminescence.

Transient absorption spectroscopy: Transient absorption spectroscopy was carried out using a home built pump-probe system. The output of a titanium:sapphire amplifier (Coherent LIBRA $\mathrm{HE}, 3.5 \mathrm{~W}, 1 \mathrm{kHz}, 100 \mathrm{fs}$ pulse duration) was split into two beams each of which was coupled into a commercial optical parametric amplifier (Coherent OPerA Solo). The first optical parametric amplifier (OPA1) was used to generate a visible pump pulse for measurements in the ps-ns time range. The second optical parametric amplifier (OPA2) was used as a seed to generate the white light continuum that was employed as the probe pulse. For measurements in the range $550-1000 \mathrm{~nm}$, a seed wavelength of $1300 \mathrm{~nm}$ was selected, and was focused into a c-cut sapphire plate $3 \mathrm{~mm}$ in thickness. For measurements in the near infrared range (1050$1600 \mathrm{~nm}$ ), a seed wavelength of $2100 \mathrm{~nm}$ was selected. To generate the NIR probe supercontinuum, a $\mathrm{YVO}_{4}$ crystal was used as the generation medium. Furthermore, a dichroic 


\section{WILEY-VCH}

mirror was placed before the sample to remove the residual idler beam $(2100 \mathrm{~nm})$ from the white-light supercontinuum. The probe pulse was focused onto the sample in a perpendicular geometry and re-collimated after the sample. It was finally dispersed on either a linear silicon photodiode array (visible range) or Peltier cooled 512 pixel InGaAs array (NIR range) and read out at $1 \mathrm{kHz}$.

Measurements in the ps-ns time range were carried out by using the visible output from OPA1 as the pump pulse, mechanically delayed with respect to the probe by use of a broadband retroreflector mounted on a variable delay stage and chopped at $500 \mathrm{~Hz}$, such that adjacent pulses representing pump on and pump off states were used to calculate $\Delta \mathrm{T} / \mathrm{T}$. Measurements in the ns-ms range employed an actively Q-switched Nd:YVO4 laser (AOT Ltd, MOPA) with a repetition rate of $500 \mathrm{~Hz}$ as the pump; a notch filter with a centre wavelength of $533 \mathrm{~nm}$ (Thorlabs) was used to remove the pump and prevent artifacts from appearing in the data.

Transient photoluminescence measurements: Transient photoluminescence data were collected using a streak camera system (Hamamatsu C4742). The solid samples were housed in a home built sample chamber under a dynamic vacuum of $10^{-5} \mathrm{mbar}$, while solution samples were housed in $1 \mathrm{~mm}$ cuvettes with all sample transfer taking place in a $\mathrm{N}_{2}$ filled glovebox. Samples were excited at $400 \mathrm{~nm}$ by the output of a commercial titanium:sapphire oscillator (Coherent Vitesse), frequency doubled in a BBO crystal.

Transmission Electron Microscopy (TEM): Films of $\sim 70 \mathrm{~nm}$ thickness were spun cast on PEDOT:PSS coated glass substrates. The films were floated off the PEDOT:PSS coated substrates in deionized water and collected on lacey carbon coated TEM grids (Electron Microscopy Sciences). TEM images were recorded in bright field mode with a microscope operating at $120 \mathrm{keV}$ (Tecnai Bio twin, FEI), using an 4k x 4k eagle CCD camera (FEI). A 100 um objective aperture was used in order to improve the contrast.

$C \& S$ mapping: FEI Company's Titan 80-300 CT TEM instrument was employed to perform the conventional TEM (CTEM), selected area electron diffraction (SAED), electron energy loss spectroscopy (EELS), and energy-filtered TEM (EFTEM) analyses of the prepared specimens. The microscope, equipped with a field-emission electron gun, was operated at the accelerating of $300 \mathrm{kV}$ during the experiments. The CTEM and SAED images are recorded on a charged coupled devices (CCD) camera of model US 4000 from Gatan, Inc. While the EELS and EFTEM analyses are carried out with a post-column energy filter also from Gatan 


\section{WILEY-VCH}

of model GIF TridiemTM. Elemental maps of sulfur and carbon are generated by selecting their S-L23 and C-K energy-edges, respectively. Moreover the socalled 3-window method was adapted to acquire these elemental maps. The entire data acquisition as well as their corresponding analysis is carried out in Gatan Microscopy Suite v.1.8.3.

Grazing Incidence X-ray Scattering (GIXS): Silicon substrates were cleaned using the ITO cleaning procedure outlined in the Device Fabrication section, except the substrates were not scrubbed with or sonicated in dilute Extran 300 detergent solution. The substrates were then coated with PEDOT:PSS using the procedure outlined in the Device Fabrication section and the PBDTTPD thin films were spin-cast using the same procedures as those used for solar cell fabrication.

GIXS experiments were performed at the Stanford Synchrotron Radiation Lightsource beamline 11-3 using a photon energy of $12.7 \mathrm{keV}$, a MAR345 image plate area detector, and an incident X-ray beam angle of $\sim 0.12^{\circ}$. During measurement, samples were kept in a helium-filled chamber to prevent beam damage and to minimize X-ray scattering due to air.

All X-ray diffraction data analysis was performed with the WxDiff software package developed by Dr. Stefan Mannsfeld. Cake segments from 70 to $110^{\circ}$ were used to determine the $\pi-\pi$ stacking peak location and $\mathrm{d}$-spacing. Peak locations were obtained using multiple peak fitting (using pseudo-Voigt peaks) and background subtraction. For all samples, cake segments were fit with flat and power law backgrounds and the appropriate number of diffraction peaks to obtain a proper fit.

\section{Supporting Information}

Supporting Information is available from the Wiley Online Library or from the author. All questions regarding the synthesis and structural characterization should be addressed to P.M. Beaujuge, while those regarding the spectroscopy and photophysics should be addressed to F. Laquai.

\section{Acknowledgements}

F.L. is very grateful to the Max Planck Society for funding the Max Planck Research Group and acknowledges support within the priority program 1355 of the Deutsche Forschungsgemeinschaft (DFG). C.D-S. is grateful to the Max Planck Society for a Research Scholarship. I.A.H. acknowledges a fellowship of the Alexander von Humboldt Foundation and the Max Planck Society via an MPG fellowship. PMB acknowledges financial support under Baseline Research Funding from King Abdullah University of Science and Technology (KAUST). The authors thank KAUST Analytical Core Labs for mass spectrometry and elemental analyses, and Sandra Seywald (MPIP - Mainz, Germany) for SEC measurements. The authors thank the Advanced Imaging and Characterization Laboratories at KAUST for 


\section{WILEY-VCH}

technical support. Portions of this research were carried out at the Stanford Synchrotron Radiation Lightsource user facility, operated by Stanford University on behalf of the U.S. Department of Energy, Office of Basic Energy Sciences.

Received: ((will be filled in by the editorial staff))

Revised: ((will be filled in by the editorial staff))

Published online: ((will be filled in by the editorial staff))

[1] a) He, Z.; Zhong, C.; Huang, X.; Wong, W.-Y.; Wu, H.; Chen, L.; Su, S.; Cao, Y., Adv.

Mater. 2011, 23 (40), 4636-4643; b) Service, R. F., Science 2011, 332 (6027), 293.

[2]

a) Chen, H.-Y.; Hou, J.; Zhang, S.; Liang, Y.; Yang, G.; Yang, Y.; Yu, L.; Wu, Y.; Li,

G., Nat.Photon. 2009, 3 (11), 649-653; b) Cabanetos, C.; El Labban, A.; Bartelt, J. A.;

Douglas, J. D.; Mateker, W. R.; Frechet, J. M.; McGehee, M. D.; Beaujuge, P. M., J. Am.

Chem. Soc. 2013, 135 (12), 4656-9; c) Small, C. E.; Chen, S.; Subbiah, J.; Amb, C. M.; Tsang, S.-W.; Lai, T.-H.; Reynolds, J. R.; So, F., Nat. Photon. 2012, 6 (2), 115-120; d) You, J.; Dou, L.; Yoshimura, K.; Kato, T.; Ohya, K.; Moriarty, T.; Emery, K.; Chen, C.-C.; Gao, J.; Li, G.; Yang, Y., Nat. Commun. 2013, 4, 1446; e) You, J.; Chen, C.-C.; Hong, Z.; Yoshimura, K.;

Ohya, K.; Xu, R.; Ye, S.; Gao, J.; Li, G.; Yang, Y., Adv. Mater. 2013, 25 (29), 3973-3978.

[3] a) Di Nuzzo, D.; Aguirre, A.; Shahid, M.; Gevaerts, V. S.; Meskers, S. C. J.; Janssen, R. A. J., Adv. Mater. 2010, 22 (38), 4321-4324; b) Etzold, F.; Howard, I. A.; Forler, N.; Cho, D. M.; Meister, M.; Mangold, H.; Shu, J.; Hansen, M. R.; Müllen, K.; Laquai, F., J. Am. Chem. Soc. 2012, 134 (25), 10569-10583; c) Nguyen, L.; Hoppe, H.; Erb, T.; Guenes, S.; Gobsch, G.; Sariciftci, N., Adv. Funct. Mater. 2007, 17 (7), 1071-1078; d) Stuart, A. C.; Tumbleston, J. R.; Zhou, H.; Li, W.; Liu, S.; Ade, H.; You, W., J. Am. Chem. Soc. 2013; e) van Duren, J. K. J.; Yang, X. N.; Loos, J.; Bulle-Lieuwma, C. W. T.; Sieval, A. B.; Hummelen, J. C.; Janssen, R. A. J., Adv. Funct. Mater. 2004, 14 (5), 425-434; f) Chen, M. S.; Niskala, J. R.; Unruh, D. A.; Chu, C. K.; Lee, O. P.; Fréchet, J. M. J., Chem. Mater. 2013; g) Liu, F.; Gu, Y.; Jung, J. W.; Jo, W. H.; Russell, T. P., J. Polym. Sci. B Polym. Phys. 2012, 50 (15), 1018-1044. 


\section{WILEY-VCH}

[4] a) Osaka, I.; Saito, M.; Koganezawa, T.; Takimiya, K., Adv. Mater. 2014, 26 (2), 3318; b) Yiu, A. T.; Beaujuge, P. M.; Lee, O. P.; Woo, C. H.; Toney, M. F.; Fréchet, J. M. J., J. Am. Chem. Soc. 2011, 134 (4), 2180-2185.

[5] Howard, I.A., Mangold, H., Etzold, F., Gehrig, D., Laquai, F.: Transient Absorption Data Analysis by Soft-Modeling. In: G. Gurzadyan, G. Lanzani, C. Soci, T.C. Sum (Ed.): Ultrafast Dynamics in Molecules, Nanostructures and Interfaces, World Scientific - Series in Optics and Photonics - Vol. 8 2014, 53-78.

[6] Albert-Seifried, S.; Friend, R. H., App. Phys. Lett. 2011, 98 (22), 223304-3.

[7] Hoke, E. T.; Vandewal, K.; Bartelt, J. A.; Mateker, W. R.; Douglas, J. D.; Noriega, R.; Graham, K. R.; Fréchet, J. M. J.; Salleo, A.; McGehee, M. D., Adv. Energy Mater. 2013, 3 (2), $220-230$.

[8] Paraecattil, A. A.; Beaupré, S.; Leclerc, M.; Moser, J.-E.; Banerji, N., J. Phys. Chem. Lett. 2012, 2952-2958.

[9] a) Albrecht, S.; Schindler, W.; Kurpiers, J.; Kniepert, J.; Blakesley, J. C.; Dumsch, I.; Allard, S.; Fostiropoulos, K.; Scherf, U.; Neher, D., J. Phys. Chem. Lett. 2012, 3 (5), 640-645; b) Clarke, T. M.; Durrant, J. R., Chem. Rev. 2010; c) Howard, I. A.; Mauer, R.; Meister, M.; Laquai, F., J. Am. Chem. Soc. 2010, 132 (42), 14866-14876; d) Jamieson, F. C.; Agostinelli, T.; Azimi, H.; Nelson, J.; Durrant, J. R., J. Phys. Chem. Lett. 2010, 3306-3310; e) Petersen, A.; Ojala, A.; Kirchartz, T.; Wagner, T. A.; Würthner, F.; Rau, U., Phys. Rev. B 2012, 85 (24), 245208.

[10] a) Shuttle, C. G.; O'Regan, B.; Ballantyne, A. M.; Nelson, J.; Bradley, D. D. C.; Mello, J. d.; Durrant, J. R., App. Phys. Lett. 2008, 92 (9), 093311; b) Etzold, F.; Howard, I. A.; Mauer, R.; Meister, M.; Kim, T. D.; Lee, K. S.; Baek, N. S.; Laquai, F., J. Am. Chem. Soc. 2011, 133 (24), 9469-9479.

[11] Piliego, C.; Holcombe, T. W.; Douglas, J. D.; Woo, C. H.; Beaujuge, P. M.; Fréchet, J. M. J., J. Am. Chem. Soc. 2010, 132 (22), 7595-7597. 


\section{WILEY-VCH}

[12] Jamieson, F. C.; Domingo, E. B.; McCarthy-Ward, T.; Heeney, M.; Stingelin, N.;

Durrant, J. R., Chem. Science 2012, 3 (2), 485-492.

[13] Rao, A.; Chow, P. C.; Gelinas, S.; Schlenker, C. W.; Li, C. Z.; Yip, H. L.; Jen, A. K.;

Ginger, D. S.; Friend, R. H., Nature 2013, 500 (7463), 435-9.

[14] Hwang, I.; Beaupre, S.; Leclerc, M.; Scholes, G. D., Chem. Science 2012, 3 (7), 22702277.

[15] a) Chen, K.; Barker, A. J.; Reish, M. E.; Gordon, K. C.; Hodgkiss, J. M., J. Am. Chem. Soc. 2013; b) Kaake, L. G.; Jasieniak, J. J.; Bakus, R. C.; Welch, G. C.; Moses, D.; Bazan, G. C.; Heeger, A. J., J. Am. Chem. Soc. 2012.

[16] (a) Jaumot, J.; Gargallo, R.; de Juan, A.; Tauler, R., A graphical user-friendly interface for MCR-ALS: a new tool for multivariate curve resolution in MATLAB. Chemometrics and Intelligent Laboratory Systems 2005, 76 (1), 101-110; (b) http://www.mcrals.info/. 


\section{WILEY-VCH}

The polymer side-chain pattern determines the efficiency of PBDTTPD:PCBM solar cells, because it changes the yield of free charges and the non-geminate recombination dynamics as demonstrated by broadband transient pump-probe spectroscopy. Tuning of the side chains leads to a doubling of the power conversion efficiency from $4 \%$ up to $8 \%$.

\section{Polymer Solar Cells}

Clare Dyer-Smith, Ian A. Howard, Clément Cabanetos, Abdulrahman El Labban, Pierre M. Beaujuge, ${ }^{*}$ Frédéric Laquai*

Interplay Between Side-Chain Pattern, Polymer Aggregation, and Charge Carrier Dynamics in PBDTTPD:PCBM Bulk-Heterojunction Solar Cells

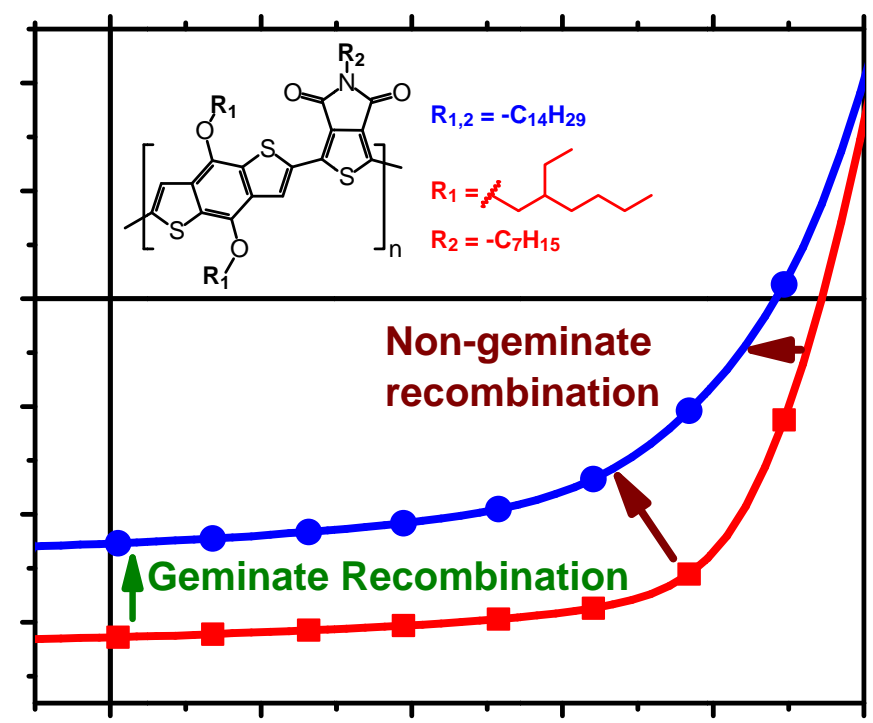

